\title{
Can regadenoson pharmacologic SPECT be performed effectively in patients with left bundle branch block or paced rhythm patients? A retrospective comparison to adenosine SPECT*
}

\author{
Gurunanthan Palani $^{1}$, Aarthee S. Karthikeyan ${ }^{2}$, Jacobsen Gordon ${ }^{2}$, Karthik Ananthasubramaniam ${ }^{2 \#}$ \\ ${ }^{1}$ Department of Internal Medicine, McLaren/Michigan State University, Flint, USA \\ ${ }^{2}$ Heart and Vascular Institute, Henry Ford Hospital, Detroit, USA \\ Email: "kananth1@,hfhs.org \\ Received 13 January 2013; revised 10 March 2013; accepted 13 April 2013 \\ Copyright (C) 2013 Gurunanthan Palani et al. This is an open access article distributed under the Creative Commons Attribution Li- \\ cense, which permits unrestricted use, distribution, and reproduction in any medium, provided the original work is properly cited.
}

\begin{abstract}
Purpose: Regadenoson (REG) is currently becoming the stress agent of choice in patients undergoing pharmacologic single photon emission computed tomography (SPECT). However, in patients with left bundle branch block (LBBB) and ventricular paced rhythm (VPR), hesitation exists amongst clinicians to use REG-SPECT due to the concern that the increased heart rate could cause false positive SPECT results. We sought to evaluate the comparability of A-SPECT and REG-SPECT in patients with LBBB and VPR. Methods: Retrospective study of 30 patients who served as their own controls. All 30 patients who underwent REG-SPECT (Grp 1) were compared to their prior A-SPECT (Grp 2) done within two years prior to REG-SPECT. Heart rate (HR) and blood pressure (BP) parameters, ECG, stress perfusion and gated variables, SPECT ischemia, and side-effects were evaluated. Statistical significance was set at $P<0.05$. Results: Grp 1 and Grp 2 were comparable in hemodynamic parameters with increase in HR and decrease in systolic and diastolic BP with administration of adenosine and REG stress agents. However, there were no significant differences found in hemodynamic parameters and II degree AV block between the groups. All normal A-SPECT were found to be normal with REG-SPECT. No differences could be found between the two groups among SPECT parameters. Muscle pain was significantly higher in REG $(\mathbf{1 0 . 0} \%$ vs. $0.0 \%, P=\mathbf{0 . 0 8 3})$ and so was the use of aminophylline $(16.7 \%$ vs. $0.0 \%, P=$
\end{abstract}

*Research grant support: Astellas pharma US Inc. (KA).

${ }^{\#}$ Corresponding author.
0.025) to relieve the side-effect. Conclusion: REGSPECT can be administered in patients with LBBB and VPR patients based on favorable and comparable hemodynamic responses and arrhythmia occurrences to A-SPECT. REG-SPECT can also be used for adequate interpretation of presence or absence of SPECT ischemia particularly in the LAD territory without any concern for false positive perfusion defects.

Keywords: Regadenoson; Single Photon Emission Computed Tomography; Left Bundle Branch Block; Ventricular Paced Rhythm; Adenosine; False Positive SPECT

\section{INTRODUCTION}

Approximately 17.6 million people in the United States are known to have coronary heart disease (CHD) [1] and more than 2.4 million people have cardiac pacemakers implanted for various clinical indications. The prevalence of left bundle branch block (LBBB) is approximately $0.2-0.8$ percent of the general population and it increases with age [2]. Ono et al. has shown in his animal experimental study that by inducing LBBB, the blood flow to the myocardial septum is decreased due to reduced contractility and increased pressure in myocardium [3]. In these patients with LBBB and ventricular paced rhythm (VPR), coronary artery disease evaluation is challenging as observed septal ischemia can be misleading. Traditional assessment such as exercise treadmill test is inconclusive due to uninterpretable electrocardiogram. Most clinicians resort to stress imaging; however, for patients with LBBB or VPR, exercise stress test with single photon emission computerized tomogramphy (SPECT) is associated with higher false posi- 
tive results due to tachycardia induced perfusion defects in the septum and are best evaluated with pharmacologic stress SPECT [4,5].

Adenosine and dipyridamole are most widely used and well known pharmacological vasodilator stress agents for patients with baseline ECG abnormality. However, these non-selective adenosine agonist stress agents act on other adenosine receptor subtypes such as $A_{1}, A_{2 B}$, and $A_{3}$ causing frequent side-effects like AV block, peripheral vasodilation, and bronchoconstriction. Hence, adenosine 2A selective pharmacological stress agent could provide adequate coronary vasodilation for SPECT and lessen/ avoid other undesirable side-effects.

Regadenoson (REG) was approved as a pharmacological stress agent for SPECT by the Food and Drug Administration in April 2008 [6]. REG is rapidly becoming the most widely used vasodilator agent in patients undergoing SPECT. It has been shown to be comparable with adenosine in diagnostic accuracy for coronary artery disease (CAD) in the ADVANCE MPI trials [7-9]. REG selectively activates A2A receptors causing rapid increase in coronary blood flow for duration of approximately $2-5$ minutes [10], which is adequate for radionuclide uptake and ideal to perform SPECT studies. The drug has 9 times more affinity for adenosine $A_{2 A}$ receptors than that for adenosine $A_{1}$ receptors and its affinity for $\mathrm{A}_{2 \mathrm{~B}}$ and $\mathrm{A}_{3}$ receptors are minimal [11]. Although, LBBB or VPR are not considered a contraindication for REG-SPECT in the package insert [12], hesitation exists amongst clinicians. This is due to the concern that increased heart rate observed in REG due to direct sympathetic stimulation could cause false positive perfusion defects in the septal area supplied by left anterior descending artery (LAD) similar to what has been previously reported with exercise SPECT in LBBB patients $[4,5]$. These early studies have led to the notion that increased heart rate is not ideal when evaluating patients with LBBB or VPR for CAD particularly with SPECT. Due to the aforementioned concerns, the 2007 ACC/AHA guidelines on stress testing suggested that pharmacologic stress testing is the preferred approach when evaluating for $\mathrm{CAD}$ in this patient subset [13].

We sought to evaluate the comparability of Adenosine-SPECT (A-SPECT) and REG-SPECT in terms of perfusion defects with particular focus on LAD distribution, hemodynamics, and overall scan interpretability in patients with LBBB and paced rhythm.

\section{METHODS}

We retrospectively identified 30 patients $(\mathrm{N}=30)$ out of 1049 patients who served as their own controls from our nuclear cardiology database. These patients were selected as the study group as they underwent a REGSPECT study for various clinical indications during
January 2009 to July 2010 but also had a clinically indicated A-SPECT study done within two years prior to REG SPECT. Patients with documented inter-current events such as myocardial infarction or any form of coronary revascularization between the two SPECT studies were excluded. Patients who underwent the study with REG were grouped as REG-SPECT (Group 1) and the variables were compared with their prior A-SPECT test results (Group 2). The average duration between A-SPECT and REG-SPECT study was 279 days. The patient's heart rate (HR), blood pressure (BP), ECG, SPECT ischemia, stress perfusion and gated variables for both SPECT studies were collected from electronic medical records. SPECT ischemia was defined as a qualitative report for ischemia with a summed difference score of $\geq 2$. Patient's hemodynamic changes, immediate major clinical events like arrhythmias, side-effects (headache and muscle pain), and use of aminophylline were reviewed. The study was approved by our institutional ethics board.

REG-SPECT Protocol: All patients underwent standard low dose rest-high dose stress SPECT acquisition with a dual head camera as per American Society of Nuclear Cardiology guidelines for SPECT acquisition specifics [14]. After rest imaging was completed with $8-10$ millicuries (mCi) of T-99 m tetrofosmin, a standard 400 microgram REG bolus was used followed by a saline flush as recommended in the package insert and approximately twenty seconds later, $25-30 \mathrm{mCi}$ of Tc-99 $\mathrm{m}$ tetrofosmin was injected intravenously and stress SPECT acquisition was done. Both rest and stress images were gated. As per our laboratory protocol, patients with resting ECG demonstrating LBBB or paced rhythm do not undergo adjunctive low level exercise to avoid any undesirable increase in HR. Vital signs, subjective symptoms, hemodynamic and electrocardiographic responses were all collected in a standard stress test worksheet. Aminophylline was used to reverse effects of REG for persistent side-effects beyond 15 minutes of completion of REG bolus. The decision to hold anti-anginal medications such as beta-blockers $12-24$ hours prior to stress imaging was left up to the discretion of the ordering physician.

\section{STATISTICAL ANALYSIS}

Continuous variables are expressed as mean (SD) and compared using the paired t-test, whereas McNemar's test for matched pairs was used for comparison of categorical variables. Statistical significance was set at $\mathrm{P}<$ 0.05 .

\section{RESULTS}

The indications for the SPECT study among both study 
groups are presented in Table 1. The most common indications for the study were chest pain and dyspnea. Baseline characteristics of the entire study group showed a higher prevalence of hypertension, diabetes, CAD, and $\mathrm{EF}<50 \%$ in this population as shown in Table 1.

Arrhythmias: Arrhythmias were found in 50.0\% of Grp 1 and Grp 2 patients. Out of 15 patients who had arrhythmias in Grp 2, one patient had first degree and three patients had second degree atrio-ventricular block which were non-statistically significant compared to none in the same patients during their subsequent REGSPECT study (Table 2).

Hemodynamics: There was a slight increase in HR and decrease in systolic and diastolic BP with administration of adenosine and REG stress agents. However, there were no significant differences in hemodynamic parameters between the groups (Table 2).

SPECT Parameters: No differences were found in the detection of ischemia between the 2 groups (Table 3 ).

Table 1. Baseline characteristics of the entire study group.

\begin{tabular}{|c|c|c|}
\hline Variable & \multicolumn{2}{|c|}{ All Patients N = 30} \\
\hline Gender-Male, n (\%) & \multicolumn{2}{|c|}{$13(43.3 \%)$} \\
\hline Race-White, n (\%) & \multicolumn{2}{|c|}{$19(63.3 \%)$} \\
\hline Age (SD), Years & \multicolumn{2}{|c|}{$73.7 \pm 8.5$} \\
\hline Weight (SD), lbs & \multicolumn{2}{|c|}{$192.2 \pm 52.5$} \\
\hline Body Surface Area (SD), $\mathrm{m}^{2}$ & \multicolumn{2}{|c|}{$1.94 \pm 0.3$} \\
\hline Smoker, n (\%) & \multicolumn{2}{|c|}{$3(10.0 \%)$} \\
\hline Asthma, n (\%) & \multicolumn{2}{|c|}{$2(6.7 \%)$} \\
\hline $\begin{array}{l}\text { Chronic Obstructive Pulmonary } \\
\text { Disease, n (\%) }\end{array}$ & \multicolumn{2}{|c|}{$3(10.0 \%)$} \\
\hline Diabetes Mellitus, n (\%) & \multicolumn{2}{|c|}{$12(40.0 \%)$} \\
\hline Hypertension, n (\%) & \multicolumn{2}{|c|}{$28(93.3 \%)$} \\
\hline Hypercholesterolemia, n (\%) & \multicolumn{2}{|c|}{$21(70.0 \%)$} \\
\hline Coronary Artery Disease, n (\%) & \multicolumn{2}{|c|}{$21(70.0 \%)$} \\
\hline Myocardial Infarction, n (\%) & \multicolumn{2}{|c|}{$10(33.3 \%)$} \\
\hline Cerebrovascular Accident, $\mathrm{n}(\%)$ & \multicolumn{2}{|c|}{$5(16.7 \%)$} \\
\hline Ejection Fraction $<50 \%, \mathrm{n}(\%)$ & \multicolumn{2}{|c|}{$11(36.7 \%)$} \\
\hline Indications: & REG-SPECT & A-SPECT \\
\hline Chest Pain & $23(76.7 \%)$ & $19(63.3 \%)$ \\
\hline Dyspnea & $4(13.3 \%)$ & $5(16.6 \%)$ \\
\hline Coronary Artery Disease & $1(3.3 \%)$ & $3(10.0 \%)$ \\
\hline Congestive Heart Failure & $1(3.3 \%)$ & - \\
\hline Pre-Operative Evaluation & $1(3.3 \%)$ & $1(3.3 \%)$ \\
\hline Abnormal EKG & - & $2(6.6 \%)$ \\
\hline
\end{tabular}

Table 2. Comparison of hemodynamic changes and arrhythmias between REG and A-SPECT.

\begin{tabular}{cccc}
\hline Variable & REG-SPECT & A-SPECT & P-value \\
\hline Pre Heart rate & $66.4 \pm 10.0$ & $67.5 \pm 8.8$ & 0.530 \\
Post Heart rate & $81.4 \pm 18.0$ & $79.3 \pm 15.6$ & 0.510 \\
$\Delta$ Heart rate & $15.0 \pm 14.6$ & $11.6 \pm 12.3$ & 0.249 \\
Pre Systolic BP & $138.3 \pm 24.6$ & $135.1 \pm 21.6$ & 0.427 \\
Post Systolic BP & $125.6 \pm 26.1$ & $120.5 \pm 18.5$ & 0.217 \\
$\Delta$ Systolic BP & $-12.8 \pm 14.9$ & $-14.6 \pm 16.0$ & 0.648 \\
Pre Diastolic BP & $76.1 \pm 10.1$ & $72.7 \pm 11.7$ & 0.274 \\
Post Diastolic BP & $66.2 \pm 9.6$ & $66.4 \pm 10.7$ & 0.942 \\
$\Delta$ Diastolic BP & $-9.9 \pm 10.9$ & $-6.3 \pm 9.5$ & 0.254 \\
Arrhythmias: & & & \\
No Arrhythmias & $15 / 30(50.0 \%)$ & $15 / 30(50.0 \%)$ & 0.739 \\
PVC & $14 / 30(46.7 \%)$ & $9 / 30(33.3 \%)$ & 0.096 \\
I Degree AV Block & $0 / 30(0.0 \%)$ & $1 / 30(3.3 \%)$ & 0.317 \\
II Degree AV Block & $0 / 30(0.0 \%)$ & $3 / 30(10.0 \%)$ & 0.083 \\
PAC/PVC & $1 / 30(3.3 \%)$ & $2 / 30(6.7 \%)$ & 0.317 \\
\hline Blood pros & & & \\
\hline
\end{tabular}

$\mathrm{BP}$-Blood pressure; $\Delta$-Change; PVC-Premature ventricular complex; AV Block-Atrio-ventricular block; PAC_-Premature atrial complex.

Table 3. Comparison of SPECT parameters between REG and A-SPECT.

\begin{tabular}{cccc}
\hline Variable & REG-SPECT & A-SPECT & P-value \\
\hline Rest EDV & $111.9 \pm 55.4$ & $104.3 \pm 45.7$ & 0.237 \\
Post EDV & $119.5 \pm 64.2$ & $110.2 \pm 45.6$ & 0.249 \\
Rest ESV & $52.0 \pm 43.4$ & $45.9 \pm 34.7$ & 0.275 \\
Post ESV & $55.9 \pm 47.3$ & $52.5 \pm 33.7$ & 0.548 \\
Rest EF & $59.3 \pm 14.2$ & $58.3 \pm 12.5$ & 0.602 \\
Post EF & $60.8 \pm 11.7$ & $56.3 \pm 11.4$ & 0.056 \\
SSS & $8.00 \pm 9.4$ & $7.97 \pm 8.2$ & 0.976 \\
SRS & $6.83 \pm 8.7$ & $6.41 \pm 7.7$ & 0.724 \\
SDS & $2.00 \pm 2.80$ & $1.90 \pm 3.6$ & 0.812 \\
TID & $1.05 \pm 0.14$ & $1.13 \pm 0.15$ & 0.125 \\
SPECT Positive & $16 / 30(53.3 \%)$ & $17 / 30(56.6 \%)$ & 0.317 \\
\hline
\end{tabular}

EDV-End diastolic volume; ESV-End systolic volume; EF-Ejection fraction; SSS-Summed stress score; SRS-Summed rest score; SDSSummed difference score; TID_-Transient ischemic dilation; SPECTSingle photon emission computerized tomography.

All normal A-SPECT studies were found normal by REG-SPECT except in one A-SPECT patient who had mild left circumflex disease on A-SPECT not seen on 
REG-SPECT (Table 4). This patient declined to have a cardiac catheterization but had a follow-up REG-SPECT which did not show any defects. In REG-SPECT group 7 patients had LAD territory defects out of which 5 previously had an A-SPECT LAD defect. Out of the 2 patients who had REG-SPECT defects but not noted in A-SPECT 1 underwent cardiac catheterization after the abnormal REG-SPECT which showed proximal LAD 60\% - 69\% and diagonal $80 \%-89 \%$. The other REG-SPECT LAD defect patient declined to have cardiac catheterization in view of her stage IV chronic kidney disease.

Tolerability: Headache ( $13.3 \%$ vs. $26.7 \%, \mathrm{P}=0.157)$ and muscle pain $(10.0 \%$ vs. $0.0 \%, \mathrm{P}=0.083)$ were the common side-effects reported in both groups. Muscle pain was reported more in REG and so, the use of aminophylline to ward off the side-effect was greater in REG-SPECT group (Table 5).

Medications: No significant differences were found in medication usage between the groups at the time of the SPECT studies (Table 5).

\section{DISCUSSION}

This retrospective study sought to evaluate the feasibility

Table 4. Comparison of perfusion defects between abnormal REG and A-SPECT patients.

\begin{tabular}{ccc}
\hline Patients with SPECT Positive & REG-SPECT & A-SPECT \\
\hline 1 & LAD & LCX \\
2 & LAD & LAD \\
3 & LAD & LAD \\
4 & PDA & PDA \\
5 & LCX & LCX \\
6 & LCX & LCX \\
7 & RCA & RCA \\
8 & LAD & LAD \\
9 & LCX & LCX \\
10 & PDA & PDA \\
11 & LAD & LAD \\
12 & LCX, PDA & PDA \\
13 & LAD & LAD \\
14 & LCX & LCX \\
15 & LCX & LCX \\
16 & LAD & RCA \\
17 & Normal & LCX \\
\hline
\end{tabular}

LAD — Left anterior descending artery; LCX—Left circumflex artery; RCA —Right coronary artery; PDA—Posterior descending artery.
Table 5. Comparison of side-effects and medication usage between REG and A-SPECT.

\begin{tabular}{cccc}
\hline Variable & REG-SPECT & A-SPECT & P-value \\
\hline Headache & $4 / 30(13.3 \%)$ & $8 / 30(26.7 \%)$ & 0.157 \\
Muscle Pain & $3 / 30(10.0 \%)$ & $0 / 30(0.0 \%)$ & 0.083 \\
Aminophylline & $5 / 30(16.7 \%)$ & $0 / 30(0.0 \%)$ & $0.025^{*}$ \\
Medication Usage: & & & \\
Aspirin & $22 / 30(73.3 \%)$ & $23 / 30(76.6 \%)$ & 0.655 \\
Clopidogrel & $6 / 30(20.0 \%)$ & $9 / 30(30.0 \%)$ & 0.257 \\
Beta-Blocker & $26 / 30(86.6 \%)$ & $24 / 30(80.0 \%)$ & 0.157 \\
Calcium Channel Blocker & $6 / 30(20.0 \%)$ & $8 / 30(26.6 \%)$ & 0.157 \\
Nitrates & $14 / 30(46.6 \%)$ & $10 / 30(33.3 \%)$ & 0.103 \\
Hydralazine & $6 / 30(20.0 \%)$ & $4 / 30(13.3 \%)$ & 0.157 \\
Statins & $24 / 30(80.0 \%)$ & $25 / 30(83.3 \%)$ & 0.564 \\
Ranolazine & $1 / 30(33.3 \%)$ & $1 / 30(33.3 \%)$ & 1.000 \\
\hline
\end{tabular}

${ }^{*}$ Statistically significant.

of effectively performing REG-SPECT in LBBB and VPR patients. Each patient served as their own control with no interim changes in clinical status or any revascularization procedure between the two SPECT studies. Thus, we were able to compare both sets of images and interpretations to address if REG-SPECT in patients with LBBB/VPR at baseline created any new perfusion defects particularly in the LAD distribution (septal area) not present in A-SPECT scans in the same patient.

Patients with LBBB and VPR are known to have higher false positive perfusion defects in septum with exercise than A-SPECT [4,5]. Jazmati et al. has shown that patients with LBBB undergoing exercise SPECT had $14 \%$ false positive test results [15]. Patients with VPR had electrocardiogram (ECG) changes similar to LBBB and false positive perfusion defects were noted in infero-posterior, inferior, and apical walls. Vaduganathan et al study performed exercise, adenosine and dobutamine in their 383 patients with LBBB and showed similar sensitivity for all these three tests but higher false positive septal defects for exercise MPI (46\%) compared with adenosine (11\%) and dobutamine (8\%) [16]. The 2007 ACC/AHA guidelines recommend vasodilator testing over exercise SPECT in LBBB or VPR patients and to avoid tachycardic scenarios including adjunctive low grade exercise with vasodilator testing [13].

Several hypotheses have been proposed to explain higher false positives seen with exercise SPECT. One hypothesis is that in LBBB, asynchronous activation of the left side of the septum which contracts late in the systole when rest of the ventricle already completed its 
contraction phase predisposes these patients to a shorter diastole during exercise stress testing from the resultant tachycardia. This will induce transient antero-septal and septal perfusion defects which can be overcome by a more uniform distribution of coronary blood flow using vasodilator imaging studies which tend not to cause the same degree of tachycardia.

We have demonstrated that the interpretation of ischemic and non-ischemic zones were comparable between REG and A-SPECT in the same patients serving as their own control. REG has tolerable hemodynamic changes without any second or third degree AV block. We did not observe significant increase in HR with REG-SPECT compared with A-SPECT resulting in any new defects compared to prior A-SPECT. Whether underlying electrical conduction issues in these patients prevents the normal tachycardic response to REG is unclear and needs further study but there appears to be no reason to avoid use of REG as a vasodilator for CAD assessment in patients with LBBB or VPR.

In our study, there were more number of patients who reported muscle pain (3/30) while undergoing REGSPECT when compared with their prior A-SPECT. The incidence of aminophylline use was also significantly higher in REG-SPECT (16.7\% Grp 1 vs. $0.0 \%$ Grp 2, P = 0.025 ) compared to $3 \%$ use of aminophylline in the REG and adenosine arms of ADVANCE MPI trials $[7,8]$. However, it should be noted that the ADVANCE MPI trials excluded patients with low ejection fraction, COPD, asthma, and significant CKD which makes generalizability of all of its observations to daily practice unclear. Thus, in unselected patients undergoing REG-SPECT it is likely that given REG's triphasic half life and persistence of minor symptoms 15 - 30 minutes after administration, aminophylline is used if side-effects are perceived as bothersome to the patient.

REG has a triphasic half life with maximal coronary hyperemic effects lasting 2 - 4 minutes [17] and its second phase lasting for $15-30$ minutes by which most of its side-effects subside. REG has a terminal half-life of $33-108$ minutes which is felt to be hemodynamically non-significant $[18,19]$. The main mechanism of action of REG is through adenosine $2 \mathrm{~A}$ receptors. The relevance of this to clinical practice in LBBB or VPR is that pharmacologic stress imaging is the main modality of CAD evaluation in all patients with this baseline ECG abnormality. Hence, REG could be an ideal choice of pharmacological stress agent if shown to be comparable to A-SPECT in this subgroup considering its selective coronary vasodilation, rapid onset and termination of action, hemodynamic safety profile and single bolus administration. Furthermore, as the incidence of new AV block is significantly lower with REG compared to adenosine [7], it may be a more desirable stress agent to use in patients with LBBB who may or may not have additional conduction system abnormalities such as 1 st degree AV block. Thus establishing the non-inferiority as well as comparable diagnostic efficacy of REG to A-SPECT in patients with LBBB or VPR is important to enable clinicians to become comfortable in using REG. Although our study is a small retrospective and not corroborated with angiographic data, it serves as a good starting point for larger confirmatory studies to demonstrate the effective use of REG in LBBB, and VPR.

\section{LIMITATIONS}

Smaller sample size due to the inclusion criteria of internal comparison and absence of coronary angiographic data to correlate SPECT findings are limitations of this study. These data were collected retrospectively from a single tertiary care center. Although HR's were higher in REG and A-SPECT group they were not significantly different between both groups in our study population to clearly establish the concept that tachycardia does not cause septal perfusion defects in REG-SPECT. The reason for lack of significant change in HR could be concomitant use of beta-blockers or underlying intrinsic conduction system issues preventing adequate HR increases.

\section{CONCLUSION}

This study shows that REG-SPECT can be safely and effectively administered in patients with LBBB and VPR patients based on hemodynamic responses, arrhythmia occurrences and can be used for adequate interpretation of presence or absence of SPECT ischemia particularly in the LAD territory without any concern for false positive perfusion defects.

\section{REFERENCES}

[1] Rosamond, W., Flegal, K., Furie, K., Go, A., Greenlund, K., Haase, N., Hailpern, S.M., Ho, M., Howard, V., Kissela, B., Kittner, S., Lloyd-Jones, D., McDermott, M., Meigs, J., Moy, C., Nichol, G., O’Donnell, C., Roger, V., Sorlie, P., Steinberger, J., Thom, T., Wilson, M. and Hong, Y. (2008) Heart disease and stroke statistics-2008 update: A report from the American heart association statistics committee and stroke statistics subcommittee. Circulation, 117, e25-e146. doi:10.1161/CIRCULATIONAHA.107.187998

[2] Hardarson, T., Arnason, A., Eliasson, G. J., Palsson, K., Eyjolfsson, K. and Sigfusson, N. (1987) Left bundle branch block: Prevalence, incidence, follow-up and outcome. European Heart Journal, 8, 1075-1079.

[3] Ono, S., Nohara, R., Kambara, H., Okuda, K. and Kawai, C. (1992) Regional myocardial perfusion and glucose metabolism in experimental left bundle branch block. Circulation, 85, 1125-1131. 


\section{doi:10.1161/01.CIR.85.3.1125}

[4] O'Keefe Jr., J.H., Bateman, T.M. and Barnhart, C.S. (1993) Adenosine thallium-201 is superior to exercise thallium-201 for detecting coronary artery disease in patients with left bundle branch block. Journal of the American College of Cardiology, 21, 1332-1338. doi:10.1016/0735-1097(93)90305-K

[5] Morais, J., Soucy, J.P., Sestier, F., Lamoureux, F., Lamoureux, J. and Danais, S. (1990) Dipyridamole testing compared to exercise stress for thallium-201 imaging in patients with left bundle branch block. Canadian Journal of Cardiology, 6, 5-8.

[6] Garnock-Jones, K.P. and Curran, M.P. (2010) Regadenoson. American Journal of Cardiovascular Drugs, 10, 65-71. doi:10.2165/10489040-000000000-00000

[7] Cerqueira, M.D., Nguyen, P., Staehr, P., Underwood, S.R. and Iskandrian, A.E. (2008) Effects of age, gender, obesity, and diabetes on the efficacy and safety of the selective a2a agonist regadenoson versus adenosine in myocardial perfusion imaging integrated advance-mpi trial results. JACC: Cardiovascular Imaging, 1, 307-316. doi:10.1016/j.jemg.2008.02.003

[8] Mahmarian, J.J., Cerqueira, M.D., Iskandrian, A.E., Bateman, T.M., Thomas, G.S., Hendel, R.C., Moye, L.A. and Olmsted, A.W. (2009) Regadenoson induces comparable left ventricular perfusion defects as adenosine: A quantitative analysis from the advance mpi 2 trial. JACC: Cardiovascular Imaging, 2, 959-968. doi:10.1016/i.jemg.2009.04.011

[9] Iskandrian, A.E., Bateman, T.M., Belardinelli, L., Blackburn, B., Cerqueira, M.D., Hendel, R.C., Lieu, H., Mahmarian, J.J., Olmsted, A., Underwood, S.R., Vitola, J. and Wang, W. (2007) Adenosine versus regadenoson comparative evaluation in myocardial perfusion imaging: Results of the advance phase 3 multicenter international trial. Journal of Nuclear Cardiology, 14, 645-658. doi:10.1016/i.nuclcard.2007.06.114

[10] Cerqueira, M.D. (2004) The future of pharmacologic stress: Selective a2a adenosine receptor agonists. American Journal of Cardiovascular Drugs, 94, 33D-42D.

[11] Gao, Z., Li, Z., Baker, S.P., Lasley, R.D., Meyer, S., Elzein, E., Palle, V., Zablocki, J.A., Blackburn, B. and Belardinelli, L. (2001) Novel short-acting a2a adenosine receptor agonists for coronary vasodilation: Inverse relationship between affinity and duration of action of a2a agonists. Journal of Pharmacology and Experimental Therapeutics, 298, 209-218.

[12] Lexiscan (regadenoson) injection. Deerfield IAPU, Inc.

[13] Fleisher, L.A., Beckman, J.A., Brown, K.A., Calkins, H., Chaikof, E., Fleischmann, K.E., Freeman, W.K., Froehlich, J.B., Kasper, E.K., Kersten, J.R., Riegel, B., Robb,
J.F., Smith Jr., S.C., Jacobs, A.K., Adams, C.D., Anderson, J.L., Antman, E.M., Buller, C.E., Creager, M.A., Ettinger, S.M., Faxon, D.P., Fuster, V., Halperin, J.L., Hiratzka, L.F., Hunt, S.A., Lytle, B.W., Nishimura, R., Ornato, J.P., Page, R.L., Tarkington, L.G. and Yancy, C.W. (2008) ACC/AHA 2007 guidelines on perioperative cardiovascular evaluation and care for noncardiac surgery: Executive summary: A report of the american college of cardiology/american heart association task force on practice guidelines (writing committee to revise the 2002 guidelines on perioperative cardiovascular evaluation for noncardiac surgery). Anesthesia \& Analgesia, 106, 685712. doi:10.1213/01/ane.0000309024.28586.70

[14] Hansen, C.L., Goldstein, R.A., Akinboboye, O.O., Berman, D.S., Botvinick, E.H., Churchwell, K.B., Cooke, C.D., Corbett, J.R., Cullom, S.J., Dahlberg, S.T., Druz, R.S., Ficaro, E.P., Galt, J.R., Garg, R.K., Germano, G., Heller, G.V., Henzlova, M.J., Hyun, M.C., Johnson, L.L., Mann, A., McCallister Jr., B.D., Quaife, R.A., Ruddy, T.D., Sundaram, S.N., Taillefer, R., Ward, R.P. and Mahmarian, J.J. (2007) Myocardial perfusion and function: Single photon emission computed tomography. Journal of Nuclear Cardiology, 14, e39-e60. doi:10.1016/j.nuclcard.2007.09.023

[15] Jazmati, B., Sadaniantz, A., Emaus, S.P. and Heller, G.V. (1991) Exercise thallium-201 imaging in complete left bundle branch block and the prevalence of septal perfusion defects. The American Journal of Cardiology, 67, 46-49. doi:10.1016/0002-9149(91)90097-5

[16] Vaduganathan, P., He, Z.X., Mahmarian, J.J. and Verani, M.S. (1998) Diagnostic accuracy of stress thallium-201 tomography in patients with left ventricular hypertrophy. The American Journal of Cardiology, 81, 1205-1207. doi:10.1016/S0002-9149(98)00091-5

[17] Lieu, H.D., Shryock, J.C., von Mering, G.O., Gordi, T., Blackburn, B., Olmsted, A.W., Belardinelli, L. and Kerensky, R.A. (2007) Regadenoson, a selective a2a adenosine receptor agonist, causes dose-dependent increases in coronary blood flow velocity in humans. Journal of $\mathrm{Nu}$ clear Cardiology, 14, 514-520. doi:10.1016/j.nuclcard.2007.02.016

[18] Gordi, T., Frohna, P., Sun, H.L., Wolff, A., Belardinelli, L. and Lieu, H. (2006) A population pharmacokinetic/ pharmacodynamic analysis of regadenoson, an adenosine a2a-receptor agonist, in healthy male volunteers. Clinical Pharmacokinetics, 45, 1201-1212. doi:10.2165/00003088-200645120-00005

[19] Aljaroudi, W., Hermann, D., Hage, F., Heo, J. and Iskandrian, A.E. (2010) Safety of regadenoson in patients with end-stage renal disease. The American Journal of Cardiology, 105, 133-135. doi:10.1016/j.amjcard.2009.08.663 